\title{
Antagonistic pleiotropy, mutation accumulation, and human genetic disease
}

\author{
Roger L. Albin \\ Department of Neurology, University of Michigan, Ann Arbor, MI 48109, USA
}

Received and accepted 22 June 1993

Key words: aging, Huntington's disease, hemochromatosis, myotonic dystrophy, Alzheimer's disease

\begin{abstract}
The antagonistic pleiotropy theory of senescence is the most convincing theoretical explanation of the existence of aging. As yet, no locus or allele has been identified in a wild population with the features predicted by the pleiotropic theory. Human genetic diseases offer the opportunity to identify potentially pleiotropic alleles/loci. Four human genetic diseases - Huntington's disease, idiopathic hemochromatosis, myotonic dystrophy, and Alzheimer's disease - may exhibit pleiotropic effects and further study of these diseases might result in the identification of pleiotropic genes causing aging. Inability to find an early life selective benefit associated with these disease-causing alleles would favor the major alternative genetic explanation for aging, the mutation accumulation theory.
\end{abstract}

\section{Introduction}

The antagonistic pleiotropy theory of senescence is the most rigorously formulated explanation of the existence of aging. Based on an idea of Medawar (1952), this theory has been systematically articulated by Williams (1957) and Hamilton (1966), and independently formulated by Wallace (1967). The antagonistic pleiotropy theory posits that some individual loci/alleles have different effects on fitness at different ages. If such a locus/allele had a positive effect on fitness at a relatively early age and a deleterious effect on fitness in older animals, the positive effect on fitness would outweigh the negative effect because of their relative positioning in life history. Such pleiotropic alleles would be favored to spread throughout populations and diminish fitness in older animals. Some corollaries of the pleiotropic theory seem to fit generally accepted facts about aging. For example, the pleiotropic theory predicts that there will be no single proximate mechanism of aging. Further, the pleiotropic theory predicts that the mechanisms of aging vary both between species and among organisms within a single species.
While theoretically compelling, it has been hard to accumulate direct evidence supporting the pleiotropic theory. No such pleiotropic allele/locus has been identified in a wild population. This is not surprising as identification of a pleiotropic allele/ locus would require a daunting combination of appropriate genetic, demographic, and life history data. A considerable body of experimental data derived from laboratory populations supports the pleiotropic theory (Sokal, 1970; Mertz, 1975; Rose \& Charlesworth, 1980, 1981a, 1981b; Rose, 1984; Luckinbill, 1984; Luckinbill \& Clare, 1985; Clare \& Luckinbill, 1985; Rose \& Graves, 1989; Hutchinson, Shaw \& Rose, 1991; Hutchinson \& Rose, 1991).

The major alternative genetic theory of aging, the mutation accumulation theory, is also based on a suggestion of Medawar (1952) and has similar dependence on the concept that negative effects of an allele/locus on overall fitness wane if the deleterious effect of the allele/locus occurs relatively late in life. Because of the weak selective forces acting against alleles/loci with deleterious effects late in life, these alleles/loci would tend to accumulate within the genome by a process akin to drift and 
ultimately come to provide a limit on life span. This idea differs from antagonistic pleiotropy in that no selective benefit is attached to these alleles/loci in earlier phases of life. It is possible that antagonistic pleiotropy and mutation accumulation could operate simultaneously to cause aging within a given population/species.

Study of human populations and their diseases may provide candidate alleles/loci for the ascertainment of pleiotropic genes or for finding evidence of mutation accumulation. A good deal is known about human demography and human diseases, and many human diseases have heritable components. For example, common adult-onset diseases such as breast cancer, atherosclerosis, and adult onset diabetes mellitus (AODM) have significant genetic components. These common disorders may not, however, prove suitable in the search for pleiotropic genes. For example, the high prevalence of atherosclerosis and AODM in contemporary Western societies is probably a result of the great changes in lifestyle that have resulted from economic/social modernization. Consequently, the negative effect of these diseases on the fitness of older humans may be an artifact of modern living conditions and not a true reflection of pleiotropic effects under historic conditions.

Another factor that limits the usefulness of some common human diseases in the search for candidate pleiotropic genes or mutation accumulation effects is the fact that modern medical therapies may have altered the natural history of some of these disorders, reducing their deleterious effects on fitness in later life. Finally, the genetics of these disorders is complex and probably involves multiple loci.

The search for candidate pleiotropic genes among human diseases should probably focus on rarer adult onset human diseases with less complicated genetics, distinctive phenotypes, and prior suggestions of some selective advantage. An ideal candidate for a human pleiotropic gene would fulfill the following criteria:

1) Adult onset, preferably past the peak reproductive years.

2) Simple genetics, preferably dominant inheritance with a high level of penetrance to maximize the demographic and life history information obtainable from pedigrees.

3) Phenotype independent or largely independent of environmental factors.
4) No effect of modern medical therapy on the natural history of the disease.

5) Evidence that the allele confers a selective advantage at an age earlier than the onset of disability secondary to the disease caused by the abnormal allele.

6) The existence of populations/pedigrees allowing the accumulation of sufficient demographic data to obtain an estimate of fitness.

While no human genetic disease fulfills all these criteria, at least four human genetic diseases fulfill some of these criteria and may be candidates for the ascertainment of pleiotropic effects.

This strategy is also useful in the search for mutation accumulation effects. Reasonable evidence that an adult onset genetic disease, especially a relatively common one, does not increase fitness relatively early in life would support Medawar's concept of mutation accumulation. The mutation accumulation theory would be supported even more strongly if several adult onset genetic diseases were examined and found to have no positive effect on fitness at a relatively early age.

While the strategy of using adult onset human genetic diseases in the search for pleiotropic or mutation accumulation effects is attractive, there are some specific limitations of working with human genetic diseases. Evaluating the fitness effects of alleles requires considering the effects of both the heterozygous and homozygous states. The phenotypic effect of homozygosity is not known for most human dominant diseases (though see the discussion of Huntington's disease below), and these diseases are the most attractive for pedigree tracing and accumulation of demographic data. Homozygotes of dominant human genetic disorders are quite rare and this may simplify analysis. For recessive disorders, evaluation of the fitness effects of heterozygosity is crucial.

Appropriate estimation of fitness is also crucial. While fitness should properly be measured as the number of progeny that survive to adulthood, those few studies that have attempted to look at the selective benefit of human disease alleles have tended to look at simple fecundity (see discussion of Huntington's disease below) or simply postulated some beneficial effect without measuring actual fitness.

Huntington's disease. Huntington's disease (HD) is a completely penetrant autosomal dominant 
neurodegenerative disease. HD is a true dominant disorder with homozygotes having the same phenotype as heterozygotes (Wexler et al., 1987). The phenotype is characterized by progressive involuntary movements, impairment of coordination, personality changes, and dementia (Folstein, 1989). Onset is usually in the third and fourth decades of life and death usually occurs $15-20$ years after diagnosis. The prevalence within the USA is estimated at 30-45/million (Folstein, 1989; Conneally, 1984). There is no therapy for HD.

The locus responsible for HD has not yet been identified but has been localized to the short arm of chromosome 4 . Analysis of widely dispersed pedigrees has revealed that the HD phenotype is invariably associated with the chromosome 4 locus. The spontaneous mutation rate for HD is very low and it has been suggested that the frequency of $\mathrm{HD}$ is maintained by some selective advantage conferred by the HD allele (Folstein, 1989; Hayden, 1981).

Clinicians who work with HD families are invariably impressed with the large size of sibships in affected families. This impression has given rise to speculation that the HD allele increases fitness by increasing fecundity (Hayden, 1981). Several studies have attempted to address this issue by comparing the fecundity of HD victims with control populations. Non-affected siblings have been used as controls with the result that HD victims are generally found to produce more offspring than their normal sibs (Hayden, 1981). Some studies, however, report that when unaffected sibs are compared to normal individuals, their fecundity has been found to be depressed (Reed \& Neel, 1959; Shokeir, 1975). Six studies have attempted to compare the fecundity of $\mathrm{HD}$ victims to that of the general population (Reed \& Neel, 1959; Shokeir, 1975; Marx, 1973; Stevens, 1975; Wallace \& Parker, 1973; Walker et al., 1983). One study showed depressed fecundity (Reed \& Neel, 1959), one showed the same level of fecundity as the general population (Wallace \& Parker, 1973), and four showed elevated fecundity as compared to the general population (Shokeir, 1975; Marx, 1973; Stevens, 1975; Walker et al., 1983). The latter four studies all indicated a significant advantage in fitness over the general population. Methodological differences and sampling bias may explain some of the difference in results between the study showing depressed fecundity and those showing increased fecundity. The study showing depression of fertility in HD victims ascertained HD cases by examining the records of large state-operated mental hospitals (Reed \& Neel, 1959). The authors of this study estimated that some of the depression of fecundity was due to premature termination of reproduction due to hospitalization. This study was conducted during the 1950s when permanent and relatively early hospitalization for disorders such as HD was a common practice. On the other hand, two studies reporting increased fecundity in HD were based on efforts to ascertain all $\mathrm{HD}$ victims within the study regions and were not so dependent on the records of institutionalized patients (Shokeir, 1975; Walker et al., 1983).

Two studies have attempted to longitudinally assess the possible contribution of the HD allele to fitness. Harper et al. (1979) were able to estimate the birth rate of HD carriers in south Wales throughout the 20th century and found that the birth rate of HD carriers has remained constant while that of the general population has declined. These results imply a reproductive advantage for the HD allele. Stine and Smith (1990) estimated the coefficient of selection for the HD allele in the Afrikaner population of South Africa by comparing probable prevalence of $\mathrm{HD}$ in the founding (and very small) population of Afrikaners with recent estimates of HD prevalence. Their analysis suggested that the HD allele carries a significant negative impact on fitness.

Some data, then, suggest that the HD allele confers a selective advantage and might fulfill the criteria for a pleiotropic allele. The studies reporting a reproductive advantage for $\mathrm{HD}$ victims should, however, be interpreted with caution. None of these studies attempted to control for socioeconomic or class variables that might affect fecundity. HD victims tend to come from lower socioeconomic levels (Reed et al., 1958; Mattson, 1974), which could independently alter demographic characteristics. In addition, all the above data provide birth rate information. Reed and Neel (1959) have suggested that children of HD victims tend to have a higher infant mortality rate, which might offset any selective advantage due to increased fecundity.

Testing of the hypothesis that the HD allele is a pleiotropic gene requires the comparison of a suitable HD population with a carefully matched control population. The best candidate population for this 
type of study would be the well studied Venezuela pedigree. This remarkably large (hundreds of individuals) pedigree has been carefully characterized by an international team of clinicians and geneticists (Young et al., 1986; Penney et al., 1990). Linkage analysis has been done for the entire pedigree and the $\mathrm{HD}$ allele carrier status is known for all members of the pedigree. It should be possible to determine the fecundity and progeny survival rates of all members of the pedigree. Development of a control population matched for economic and demographic variables would permit a test of the hypothesis that the HD allele is a pleiotropic senescence gene.

Idiopathic hemochromatosis. Idiopathic hemochromatosis $(\mathrm{IH})$ is an autosomal recessive inherited disorder of iron metabolism (Bothwell, Charlton \& Motulsky, 1983). IH is remarkably common. Approximately $10 \%$ of the population of North America and Europe are heterozygous for the $\mathrm{IH}$ allele, and the prevalence of homozygotes is approximately $0.3 \%$. Penetrance appears to be complete or nearly complete in men and incomplete in women. Most IH victims develop clinical symptoms between the ages of 40 and 60 (Finch \& Finch, 1955; Milder et al., 1980). IH victims suffer from progressive deterioration in liver, endocrine pancreatic, cardiac, and pituitary function. The latter can include gonadal failure (Adams, Kertesz \& Valberg, 1991; Finch \& Finch, 1955; Milder et al., 1980).

The locus responsible for $\mathrm{IH}$ is found on chromosome 6 and is closely linked to the HLA complex. The molecular defect is unknown, but the pathophysiology of $\mathrm{IH}$ is well characterized and the pathologic consequences of $\mathrm{IH}$ result from excessive gastrointestinal absorption of iron. The human capacity to excrete iron is limited and body iron stores are regulated in large part by intestinal iron absorption. In $\mathrm{IH}$, iron absorption is greatly increased. When normal iron storage capacity is saturated, iron is deposited in abnormal locations with resulting organ dysfunction. Because of menstruation, pregnancy, and lactation, women experience greater normal losses of iron than men. The greater demand for iron faced by women probably accounts for the reduced penetrance of $\mathrm{IH}$ in women.

The historic prognosis of $\mathrm{IH}$ was dismal, with most patients dying within months of diagnosis
(Milder et al., 1980). Medical therapy has had a significant impact on the natural history of $\mathrm{IH}$ (Milder et al., 1980; Adams, Speechley \& Kertesz, 1991). Insulin therapy for diabetes secondary to IH prolongs life expectancy and institution of regular phlebotomy prior to the development of organ impairment can result in normal life expectancy.

The high frequency of the $\mathrm{IH}$ allele has led to speculation that it is the result of a selective advantage. The suggested advantage is amelioration of iron deficiency anemia by enhanced intestinal iron absorption (Rotter \& Diamond, 1987; Motulsky, 1979). Iron deficiency anemia is common in industrialized countries and very common in developing nations where diets are often poor in bioavailable iron. IH homozygosity would be especially beneficial to women, who have greater demands for iron than men.

If IH could be proven to have a positive selective effect, then the IH allele would satisfy criteria for a pleiotropic gene. In addition, because of its possible differential benefits/deleterious effects in men and women, IH might be an example of a sexually antagonistic gene (Rice, 1992). Identifying IH homozygotes is relatively straightforward. A combination of serum iron studies and histocompatibility antigen typing can presently identify IH homozygotes (Dadone et al., 1982; Borecki et al., 1990). In the future, cloning of the IH locus will permit definitive ascertainment of $\mathrm{IH}$ allele heterozygotes and homozygotes. While medical therapy has altered the natural history of $\mathrm{IH}$, it should still be possible to evaluate the potential selective benefits of $\mathrm{IH}$.

One approach would be to survey a population for $\mathrm{IH}$ and determine if iron deficiency anemia is less common among IH homozygotes than among a set of matched controls. This approach should be used in a region where iron deficiency is common and will be possible only after the IH locus has been cloned. At present, IH homozygotes are identified by serum iron studies showing evidence of increased iron stores. By definition, this criterion would exclude IH homozygotes with normal or decreased iron stores and introduce significant sampling bias into the data. Another approach would be to compare the actual fitness of IH homozygotes with an appropriate control population. IH has been extensively studied in Utah (Kravitz et $a l ., 1979)$, where a relatively stable population and the extensive genealogical records maintained by 
the Church of the Latter Day Saints permit good ascertainment of pedigrees and could allow development of appropriately matched controls.

Estimating the fitness of IH allele heterozygotes would also be crucial but more difficult. IH allele heterozygotes have a mildly increased ability to absorb iron (Cartwright, 1979) and the high prevalence of the $\mathrm{IH}$ allele could be maintained by some selective advantage accruing to the heterozygotes. Identification of $\mathrm{IH}$ allele heterozygotes for a study of heterozygote fitness requires molecular genotyping and presupposes molecular identification of the IH locus.

Myotonic dystrophy. Myotonic dystrophy (MyD) is the most common form of adult-onset muscular dystrophy with an estimated prevalence of 3-5/ 100,000 (Brooke, 1986). MyD is an autosomal dominant disorder with variable expression affecting many organs. The effect of homozygosity is unknown. Myotonia (impaired relaxation of muscles following contraction) and progressive skeletal muscle degeneration are hallmarks of MyD. Other common features include cataracts, endocrine disorders including diabetes and gonadal dysfunction, cardiac arrhythmias, and mild mental retardation. Onset of symptoms often occurs in the young adult years, but in many cases the disease may not become symptomatic until considerably later in life. MyD does occur in children and the prognosis of these unfortunate children is particularly poor. Cardiac dysrhythmias and muscular weakness (which often impair swallowing and pulmonary function) predispose patients to early death. In common with a small number of other genetic diseases, $\mathrm{MyD}$ exhibits the phenomenon of 'anticipation' in which the phenotype appears earlier and with greater severity in succeeding generations. A typical pedigree might have a grandparent with cataracts and few other detectable abnormalities, an obviously affected parent in his or her thirties, and a severely affected child. There is no therapy for MyD.

Recent studies have identified the affected locus in MyD on chromosome 19 (Aslanidis et al., 1992; Buxton et al., 1992; Harley et al., 1992). This locus encodes a protein with considerable homology to known protein kinases, enzymes that regulate the activity of other proteins. The basis for the defect in MyD is insertion of unstable trinucleotide repeats. The length of these insertions can increase in suc- ceeding generations and the increasing length correlates with increasing severity of the clinical manifestations of MyD found in succeeding generations. Other human genetic diseases exhibiting anticipation also have unstable trinucleotide insertions.

MyD has a low spontaneous mutation rate and this fact has given rise to speculation that the prevalence of MyD is maintained by some selective advantage. This speculation has been bolstered by recent molecular data. The $\mathrm{MyD}$ allele is in very strong linkage disequilibrium with closely linked markers (Harley et al., 1992; Harley et al., 1991). The linkage data suggest that virtually all $\mathrm{MyD}$ cases are descended from a single mutation, a surprising fact in view of the significant impairments that usually result from MyD.

It may be possible to assess the fitness of the MyD allele by surveying MyD patients in the Saguenay region of Quebec, where MyD has the highest known prevalence in the world, estimated at 189/100,000 (Mathieu, De Braekeleer \& Prevost, 1990). All cases within this region descend from a single couple who settled in Quebec in 1657 and the MyD allele has been passed over 10-14 generations. This extended pedigree has been carefully reconstructed. The biologic fitness of the MyD allele has been determined by comparing the fitness of contemporary MyD patients with an appropriate control population. To obtain a longitudinal evaluation of the fitness of the MyD allele, it might be possible to compare the historic fitness of individuals within the MyD pedigree with a control historical population.

Alzheimer's disease. Alzheimer's disease (AD) is a common neurodegenerative disease and a tantalizing candidate for a pleiotropic senescence gene and for evaluation of mutation accumulation effects. $\mathrm{AD}$ is primarily a disease of the elderly and prevalence increases with age. As many as $15 \%$ of individuals over age 65 may suffer from $\mathrm{AD}$ and the prevalence is much higher among individuals older than 80 . The major manifestation of $A D$ is progressive dementia, often accompanied by dysphasia and dyspraxia.

Several lines of evidence suggest a substantial genetic component in AD. Epidemiological studies aimed at revealing environmental factors contributing to $\mathrm{AD}$ have not produced convincing results. Rare cases of $\mathrm{AD}$ occur at a relatively early age, 
and in many of these cases there is clear evidence of autosomal dominant inheritance. In typical $\mathrm{AD}$ cases, the late onset of disease prevents the ascertainment of inheritance patterns by conventional pedigree tracing and linkage analysis. Recent studies of typical $\mathrm{AD}$, however, have found that there is a high incidence of dementia in first degree relatives of AD patients (Breitner, 1991). Breitner (1991) has recently summarized the literature examining the incidence of dementia in first degree relatives of $\mathrm{AD}$ victims and argues convincingly that $\mathrm{AD}$ is inherited in an autosomal dominant fashion with age dependent penetrance and maximum cumulative risk of developing $\mathrm{AD}$ around age 90.

Assuming that the majority of $\mathrm{AD}$ is genetic in etiology, $\mathrm{AD}$ would be among the most prevalent of human genetic diseases. It is possible that there may be considerable genetic heterogeneity underlying the $\mathrm{AD}$ phenotype. Linkage studies of early onset pedigrees have identified three different loci responsible for $\mathrm{AD}$ (Goate et al., 1991; PericakVance et al., 1991; St. George-Hyslop et al., 1987; Tanzi et al., 1987; Van Broeckhoven, 1987). Whether genetic heterogeneity exists in typical $\mathrm{AD}$ can only be determined after molecular genetic identification of the responsible gene(s).

If $\mathrm{AD}$ is among the most prevalent of human genetic diseases, its high frequency might result from some selective advantage conferred by alleles causing $\mathrm{AD}$. The fact that $\mathrm{AD}$ achieves maximum penetrance late in life makes it an intriguing candidate for ascertainment of pleiotropic gene effects. Up to this point, there have been no suggestions that AD-causing alleles confer a selective advantage. The lack of speculation about the possible selective advantages of $\mathrm{AD}$ alleles is probably a result of the fact that most clinicians and scientists interested in $\mathrm{AD}$ have not thought of it as a genetic disease. One study of the incidence of dementia among first degree relatives of $\mathrm{AD}$ victims found also that $\mathrm{AD}$ victims tended to have larger numbers of children than controls (Fitch, Becker \& Heller, 1988) though this difference was not statistically significant (N. Fitch, personal communication).

AD-causing alleles are also excellent candidates for mutation accumulation effects. If there was no reasonable evidence that $\mathrm{AD}$-causing alleles had selective benefits at any age, then AD-causing alleles would be a prime example of mutation accumulation.
Evaluation of the effect of $\mathrm{AD}$-causing alleles would seem to be relatively straightforward. $\mathrm{AD}$ patients could be identified at autopsy or by epidemiological survey and their fitness compared with a matched set of controls. Alternatively, when the genetic defect(s) underlying typical AD are discovered, it should be possible to compare the fitness of those carrying the allele(s) with a control population. Molecular identification of loci associated with $\mathrm{AD}$ may also give clues to the possible effects on fitness of alleles causing $\mathrm{AD}$.

Conclusions. While none of the diseases discussed above fulfills the criteria of a pleiotropic gene, there is suggestive evidence of pleiotropic effects, and further study of these disorders might lead to proof of the existence of a pleiotropic gene in a wild population. The use of human disease populations is a plausible way to ascertain the existence of negative pleiotropy or mutation accumulation and has significant advantages over the use of nonhuman populations where appropriate demographic and genetic data may be hard to obtain. Negative results regarding selective benefits associated with disease-causing alleles would not disprove the pleiotropic theory, but a positive result would provide powerful evidence in favor of the pleiotropic gene theory of senescence. Negative results regarding selective benefits of disease alleles, however, would provide evidence favoring the mutation accumulation theory.

\section{Acknowledgements}

Supported by grant NS01300 from the National Institute of Neurological Disease and Stroke. I thank the anonymous reviewers for their helpful criticisms.

\section{References}

Adams, P. C., A. E. Kertesz \& L. S. Valberg, 1991. Clinical presentation of hemochromatosis: a changing scene. Am. J. Med. 90: 445-449.

Adams, P. C., M. Speechley \& A. E. Kertesz, 1991. Long-term survival analysis in hereditary hemochromatosis. Gastroenterology 101: 368-372.

Aslanidis, C., G. Jansen, C. Amemiya, G. Shutler, M. Mahadevan, C. Tsilfidis, C. Chen, J. Alleman, N. G. M. 
Wormskamp, M. Vooijs, J. Buxton, K. Johnson, H. J. M. Smeets, G. G. Lennon, A. V. Carrano, R. G. Korneluk, B. Wieringa \& P. J. de Jong, 1992. Cloning of the essential myotonic dystrophy region and mapping of putative defect. Nature 355: 548-551.

Borecki, I. B., G. M. Lathrop, G. E. Bonney, J. Yaouang \& D. C. Rao, 1990. Combined segregation and linkage analysis of genetic hemochromatosis using affection status, serum iron, and HLA. Am. J. Hum. Genet. 47: 542-550.

Bothwell, T. H., R. W. Charlton \& A. G. Motulsky, 1983. Hemochromatosis, pp. 1269-1298 in The Metabolic Basis of Inherited Disease, edited by J. B. Stanbury, J. B. Wyngaarden, D. S. Frederickson, J. L. Goldstein \& M. S. Brown. McGraw-Hill, New York.

Breitner, J. C., 1991. Clinical genetics and genetic counseling in Alzheimer Disease. Ann. Intern. Med. 115: 601-606.

Brooke, M. H., 1986. A Clinician's View of Neuromuscular Diseases. Williams \& Wilkins, Baltimore, Maryland.

Buxton, J., P. Shelbourne, J. Davies, C. Jones, T. Van Tongeren, G. Aslanidis, P. de Jong, G. Jansen, M. Anvret, B. Riley, R. Williamson \& K. Johnson, 1992. Detection of an unstable fragment of DNA specific to individuals with myotonic dystrophy. Nature 355: 547-548.

Cartwright, G. E., 1979. Hereditary hemochromatosis: phenotypic expression of the disease. New England Journal of Medicine 301: 175-179.

Clare, M. J. \& L. S. Luckinbill, 1985. The effects of geneenvironment interaction on the expression of longevity. Heredity 55 : 19-29.

Conneally, P. M., 1984. Huntington's disease: genetics and epidemiology. American Journal of Human Genetics 36: 506526.

Dadone, M. M., J. P. Kushner, C. Q. Edwards, D. T. Bishop \& M. H. Skolnick, 1982. Hereditary hemochromatosis. Analysis of laboratory expression of the disease by genotype in 18 pedigrees. Am. J. Clin. Pathol. 78: 196-207.

Finch, S. C. \& C. A. Finch, 1955. Idiopathic hemochromatosis, an iron storage disease. Medicine 34: 381-430.

Fitch, N., R. Becker \& A. Heller, 1988. The inheritance of Alzheimer's disease: a new interpretation. Ann. Neurol. 23: 14-19.

Folstein, S. E., 1989. Huntington's Disease, A Disorder of Families. The Johns Hopkins University Press, Baltimore, Maryland.

Goate, A., M. C. Chartier-Harlin, M. Mullan, J. Brown, F. Crawford, L. Fidani et al., 1991. Segregation of a missense mutation in the amyloid precursor protein gene with familial Alzheimer's disease. Nature 349: 704-706.

Hamilton, W. D., 1966. The moulding of senescence by natural selection. Journal of Theoretical Biology 12: 12-45.

Harley, H. G., J. D. Brook, J. Floyd, S. A. Rundle, S. Crow, K. V. Walsh, M. C. Thibault, P. S. Harper \& D. J. Shaw, 1991. Detection of linkage disequilibrium between the myotonic dystrophy locus and a new polymorphic DNA marker. Am. J. Hum. Genetics 49: 68-75.

Harley, H. G., J. D. Brook, S. A. Rundle, S. Crow, W. Reardon, A. J. Buckler, P. S. Harper, D. E. Housman \& D. J. Shaw, 1992. Expansion of an unstable DNA region and phenotypic variation in myotonic dystrophy. Nature 355: 545-546.

Harper, P. S., D. A. Walker, A. Tyler, R. G. Newcombe \& K.
Davies, 1979. Huntington's chorea: The basis for long-term prevention. Lancet II: $346-349$.

Hayden, M. R., 1981. Huntington's Chorea. Springer-Verlag, Berlin.

Hutchinson, E. W. \& M. R. Rose, 1991. Quantitative genetics of postponed aging in Drosophila melanogaster. I. Analysis of outbred populations. Genetics 127: 719-727.

Hutchinson, E. W., A. J. Shaw \& M. R. Rose, 1991. Quantitative genetics of postponed aging in Drosophila melanogaster. II. Analysis of selected lines. Genetics 127: 729-737.

Kravitz, K., M. Skolnick, C. Cannings, D. Carmelli, B. Baty, B. Amos, A. Johnson, N. Mendell, C. Edwards \& G. Cartwright, 1979. Genetic linkage between hereditary hemochromatosis and HLA. Am. J. Hum. Genet. 31: 601-619.

Luckinbill, L. S. \& M. J. Clare, 1985. Selection for life span in Drosophila melanogaster. Heredity 55: 9-18.

Luckinbill, L. S., R. Arking, M. J. Clare, W. C. Cirocco \& S. A. Buck, 1984. Selection for delayed senescence in Drosophila melanogaster. Evolution 38: 996-1003.

Marx, R. N., 1973. Huntington's chorea in Minnesota. Advances in Neurology 1: 237-243.

Mathieu, J., M. De Braekeleer \& C. Prevost, 1990. Genealogical reconstruction of myotonic dystrophy in the Saguenay-LacSaint-Jean area (Quebec, Canada) Neurology 40: 839-842.

Mattson, B., 1974. Huntington's chorea in Sweden. II. Social and clinical data. Acta Psychiatrica Scandinavica Supplementum 255: 221-235.

Medawar, P. B., 1952. An Unsolved Problem in Biology. H. K. Lewis, London.

Mertz, D. B., 1975. Senescent decline in flour beetle strains selected for early adult fitness. Physiological Zoology 48: $1-23$.

Milder, M. S., J. D. Cook, S. Stray \& C. A. Finch, 1980. Idiopathic hemochromatosis, an interim report. Medicine 59: 34-49.

Motulsky, A. G., 1979. Letter: Genetics of hemochromatosis. New England Journal of Medicine 301: 1291.

Penney, J. B., Jr., A. B. Young, I. Shoulson, S. Starosta-Rubenstein, S. R. Snodgrass, J. Sanchez-Ramos, M. Ramos-Arroyo, F. Gomez, G. Penchaszadeh, J. Alvir et al., 1990. Huntington's disease in Venezuela: 7 years of follow-up on symptomatic and asymptomatic individuals. Mov. Disord. 5: 93-99.

Pericak-Vance, M. A., J. L. Bebout, P. C. Gaskell, L. H. Yamaoka, W. Y. Hung, M. H. Alberts et al., 1991. Linkage studies in familial Alzheimer's disease: evidence for chromosome 19 linkage. Am. J. Human. Genet. 48: 1034-1050.

Reed, T. E., J. H. Chandler, E. M. Hughes \& R. T. Davidson, 1958. Huntington's chorea in Michigan. 1. Demography and genetics. Am. J. Hum. Genet. 10: 201-225.

Reed, T. E. \& J. V. Neel, 1959. Huntington's chorea in Michigan. 2. Selection and mutation. Am. J. Hum. Genet. 11: 107-136.

Rice, W. R., 1992. Sexually antagonistic genes: Experimental evidence. Science 256: 1436-1438.

Rose, M. R., 1984. Laboratory evolution of postponed senescence in Drosophila melanogaster. Evolution 38: 1004 1010.

Rose, M. R. \& B. Charlesworth, 1980. A test of evolutionary theories of senescence. Nature 287: 141-143. 
Rose, M. R. \& B. Charlesworth, 1981a. Genetics of life history in Drosophila melanogaster I. Sib analysis of adult females. Genetics 97: 173-186.

Rose, M. R. \& B. Charlesworth, 1981b. Genetics of life history in Drosophila melanogaster II. Exploratory selection experiments. Genetics 97: 187-196.

Rose, M. R. \& J. L. Graves, 1989. What evolutionary biology can do for gerontology. J. Gerontol. 44: B27-29.

Rotter, J. I. \& J. M. Diamond, 1987. News and Views: What maintains the frequencies of human genetic diseases? Nature 329: 289-290.

Shokeir, M. H. K., 1975. Investigations on Huntington's disease in the Canadian prairies. II. Fecundity and fitness. Clin. Genet. 11: 349-353.

Sokal, R. R., 1970. Senescence and genetic load: Evidence from tribolium. Science 167: 1733-1734.

St. George-Hyslop, P. H., R. E. Tanzi, R. J. Polinsky, J. L. Haines, L. Nee, P. C. Watkins et al., 1987. The genetic defect causing familial Alzheimer's disease maps on chromosome 21. Science 235: 885-890.

Stevens, D. L., 1981. Quoted in Hayden, M. R. Huntington's Chorea, Springer-Verlag, Berlin.

Stine, O. C. \& K. D. Smith, 1990. The estimation of selection coefficients in Afrikaners: Huntington disease, porphyria variegata, and lipoid proteinosis. Am. J. Hum. Genet. 46: $452-458$.
Tanzi, R. E., P. H. St. George-Hyslop, J. L. Haines, R. J. Polinsky, L. Nee, J. F. Foncin et al., 1987. The genetic defect in familial Alzheimer's disease is not tightly linked to the amyloid beta-protein gene. Nature 329: 156-157.

Van Broeckhoven, C., A. M. Genthe, A. Vendenberghe, B. Horsthemke, H. Bakchovens, P. Raeymaekers et al., 1987. Failure of familial Alzheimer's disease to segregate with the A4-amyloid gene in several European families. Nature 329: 153-155.

Walker, D. A., P. S. Harper, R. G. Newcombe \& K. Davies, 1983. Huntington's chorea in South Wales: mutation, fertility, and genetic fitness. J. Med. Genetics. 20: 12-17.

Wallace, D. C., 1967. The inevitability of growing old. Journal of Chronic Diseases 20: 475-486.

Wallace, D. C. \& N. Parker, 1973. Huntington's chorea in Queensland: The most recent story. Advances in Neurology 1: 223-236.

Wexler, N. S. et al., 1987. Homozygotes for Huntington's disease. Nature 326: 194-197.

Williams, G. C., 1957. Pleiotropy, natural selection, and the evolution of senescence. Evolution 11: 398-411.

Young, A. B. et al., 1986. Huntington's disease in Venezuela: Neurologic features and functional decline. Neurology 36: 244-249. 